\title{
Etablir de nouveaux cadres de référence
}

Version française

Tejaswini Niranjana

\section{(2) OpenEdition \\ Journals}

Édition électronique

URL : http://journals.openedition.org/transtexts/180

DOI : $10.4000 /$ transtexts. 180

ISSN : 2105-2549

Éditeur

Gregory B. Lee

Édition imprimée

Date de publication : 1 mai 2006

Pagination : 52-54

ISSN : 1771-2084

Référence électronique

Tejaswini Niranjana, «Etablir de nouveaux cadres de référence », Transtext(e)s Transcultures 跨文本跨 文化 [En ligne], 1 | 2006, mis en ligne le 13 septembre 2009, consulté le 19 avril 2019. URL : http:// journals.openedition.org/transtexts/180; DOI : 10.4000/transtexts.180 


\section{Developing \\ Alternative Frames of Reference \\ By Tejaswini Niranjana}

Contemporary globalization processes often obscure older historical lines of connection across the continents. My recent work has focused on the cultural practices of people of Indian origin in the Eastern Caribbean, in Trinidad. I argue that we in India need to understand this subaltern diaspora (1845-1917) of indentured labourers as the illegitimate doubles of modern Indians in India.

Here is a brief account of some of the methodological issues and theoretical questions raised by the project, speculating as to the implications of these for how we think about contexts other than our own but with similar geopolitical locations.

My main effort has been to conceptualise frameworks for comparative research across Southern contexts and contribute to developing alternative frames of reference. The method has been broadly interdisciplinary, drawing on literary studies, feminist theory, anthropology, musicology, film studies, all of which I bring to the analysis of cultural practice.

The tensions of the project have lain in my ambiguous relationship with (a) a region - the Caribbean - which initially came before me as an object of ethnographic inquiry with all its attendant anxieties, and on which I had to learn to bring to bear the sorts of critical post-Orientalism questions that I was asking in my own postcolonial context, and with (b) a subject - "the Indian in the Caribbean" - whose history and even future may not have much in common with my own. Yet, I have argued, thinking about this region and this subject may well alter the ways by which India Indians conceptualise issues of their cultural identity. Given the historical linkages between India and the Caribbean, the comparative research project detailed in this book has had to take on the challenge of not merely pointing out similarities and differences but investigating how each location is marked by the other.

What my project has tried to do primarily is to displace Western modernity as the singular legitimating pole of comparison for analyses of Southern societies. Noting that all scholarship is comparative by definition, and that the implicit point of reference is bound to be the West (although imagined differently in each context), I began by making an argument for alternative frameworks of analysis which might strain against the inherent asymmetries of the comparativist project.

The specific undertaking has been to look at the invocations of Indianness in the Caribbean. The perspective from which these invocations have been examined was one of an Indian from the subcontinent in South Asia, halfway across the world, an inhabitant of the region that was Columbus' original destination on the journey which "discovered", and mistakenly named, the (West) Indies. The historical connections between India and the Caribbean are often consigned to the marginal notes in the story of Empire. A central argument in my project was that focussing on these obscure connections would produce a very different narrative 
than the conventional one about the formations of modernity in both India and Trinidad. While the invocation or mobilisation of "India" in these two Southern locations clearly has divergent histories, I would argue that in looking at the histories together we can throw new light on the meanings and resonances of that imagined cultural space.

The focus of the project's comparative endeavour has been the question of women, concentrating on the representations and deployment of that category rather than simply talking about empirical women. I approach "Indian women" in many diverse ways: in rethinking the history of indentureship in relation to the campaigns of early twentieth century Indian nationalism, and consequently arguing for a new perspective on the making of normative Indian femininity; in looking at women of Indian origin as producers and performers of an exciting new kind of Caribbean music; in working through the historical deployment of the exotic Indian female in calypso and soca, or in re-examining that figure in contemporary Trinidadian cultural politics; and in exploring how Trinidadian music of all genres draws on the Hindi film industry in India. The project tries to establish implicit connections between the disavowal of the subaltern Indian diaspora by India and the mobilisation of India in the Caribbean, emphasising once again the centrality of notions of the "Indian woman" to both sets of processes.

The other thread running through the project is the emphasis on popular music as a key site of cultural politics. While retaining the focus on the shaping of "Indian women" and the fashioning of "Indian" identities, I seek to explore a range of musical practices in Trinidad - from chutney, calypso and soca to chutney-soca, Hindi film songs and rapso - with a view to analyzing how they demarcate the musical arena where these identities are socially performed.

The general argument of the project is that the discourses and practices of nationalist modernity, and the formation of modern subjectivities, in India and Trinidad can be understood differently by repositioning the frame of analysis. Such a repositioning, I suggest, can be achieved through a double strategy: an investigation of the multiple processes by which "subaltern" migrants from India are disavowed in past and present in their country of origin, and an inquiry into the contemporary cultural practices implicating the descendants of these migrants in their current home.

During my research in Trinidad, I was fascinated by the identities that come to be called "Indian" in the Caribbean. Often, it became clear that those who participated in the production of these identities saw them as integral to a profoundly "local" situation, with no reference to any distant homeland. Although India in the early twenty-first century is participating in new forms of globalization, when my research started in the early 1990s this process was not yet fully underway. As one anticipated the formation of new hegemonies and wondered what sort of critical interventions might be shaped, one looked back to an earlier phase of globalization - that inspired by Empire - to track the migration and transformation of bodies and cultural practices, and to speculate on what kind of subject formation the unhousing and re-housing might enable. Geographic and historical displacements, as my project tries to demonstrate, have formed uniquely local identities in the Caribbean.

A few years ago, I was returning home from Trinidad, and had boarded a BWIA plane to New York en route to Bombay. I fell into conversation with an elderly Afro-Trinidadian woman who asked where I was headed. India, I said. And why are you going there, she wanted to know. I live there, I replied. And how is it, she asked, that you come to be living so far away from home? 


\section{Etablir de nouveaux cadres de référence}

Les processus de mondialisation contemporains masquent bien souvent les liens historiques anciens existant entre les continents. Récemment mes recherches se sont concentrées sur les pratiques culturelles des populations d'origine indienne vivant à Trinité dans les Petites Antilles. Je dirais que nous, en Inde, nous nous devons de voir dans cette diaspora subalterne (1845-1917) de travailleurs asservis, l'autre illégitime des Indiens modernes vivant en Inde. Voici, en bref, une description des problèmes méthodologiques et théoriques soulevés par un tel projet, ainsi que de leur implication dans notre manière de penser aux contextes qui ne sont pas les nôtres tout en occupant des situations géopolitiques similaires.

J'ai concentré mes efforts sur la conceptualisation de cadres pour la recherche comparative dans les contextes du Sud et sur le développement de nouveaux cadres de référence. La méthode employée était largement interdisciplinaire, s'inspirant des études littéraires, de la théorie féministe, de l'anthropologie, de la musicologie, des études filmiques, que j'inclus à l'analyse des pratiques culturelles.

Les tensions du projet vinrent de l'ambiguïté de ma propre relation avec a) une région - les Antilles - qui m'était tout d'abord apparue comme un objet d'étude ethnographique avec toute l'anxiété que cela comporte, et sur laquelle j'ai dû apprendre à poser les mêmes questions que celles que je posais dans mon propre contexte postcolonial et avec b) un sujet - «l'Indien dans les Antilles» - dont l'histoire, et probablement même le futur, n'avaient pas grand-chose en commun avec les miens. Pourtant, je maintiens que de penser à cette région et à ce sujet peut changer la manière dont les Indiens d'Inde conceptualisent les questions de leur identité culturelle. Etant donné les liens culturels entre l'Inde et les Antilles, le projet de recherche comparative élaboré dans ce livre a dû répondre au défi non seulement d'exposer les différences et similitudes mais aussi de rechercher comment chaque endroit est marqué par l'autre.

Ce que mon projet a tout d'abord tenté de faire fut de déloger la modernité occidentale en tant que point légitimant singulier de comparaison dans l'analyse des sociétés du Sud. Partant du principe que toute érudition est par définition comparative, et que le point implicite de référence est nécessairement l'Ouest (bien qu'imaginé différemment dans chaque contexte), j'ai commencé à prôner les développement de nouveaux cadres d'analyse qui pourraient forcer les asymétries inhérentes au projet comparatiste.

La tâche spécifique a consisté à examiner les invocations de l'indianité dans les Antilles.

La perspective adoptée pour examiner ces invocations était celle d'une Indienne du sous continent en Asie du Sud, de l'autre côté du monde, une habitante d'une région qui était la destination première de Christophe Colomb lors du voyage qui «découvrit» pour les nommer 
à tort par la suite, les Indes occidentales. Les relations entre l'Inde et les Antilles sont souvent reléguées dans les notes en marge de l'histoire de l'Empire. L'un des principaux arguments de mon projet était que de se concentrer sur ces relations obscures apporterait un narratif différent du narratif traditionnel sur les formations de la modernité à la fois en Inde et à Trinité. Alors que l'invocation ou mobilisation de l' "Inde " a manifestement des histoires divergentes dans ces deux emplacements du Sud, il me semble qu'en étudiant ensemble leurs histoires nous pouvons apporter un nouvel éclairage aux significations et aux résonances de cet espace culturel imaginé.

Le projet comparatif s'est tout particulièrement concentré sur la question des femmes, des représentations et du déploiement de cette catégorie, plutôt que de simplement parler des femmes empiriques. J'approche « les femmes indiennes » de diverses manières :en repensant l'histoire du contrat synallagmatique en rapport avec les campagnes du nationalisme indien du début du vingtième siècle, et par conséquence en prônant une nouvelle perspective dans la fabrication d'une féminité indienne normative ; en étudiant les femmes d'origine indienne productrices et interprètes d'une nouvelle et fascinante musique antillaise ; en examinant le déploiement historique de la femme indienne exotique dans la calypso et la soca, ou en la ré-examinant dans le contexte politique contemporain de Trinité et en explorant la façon dont la musique de Trinité, tous genres confondus, emprunte à l'industrie du film Hindi en Inde. Ce projet tente d'établir des liens implicites entre le désaveu de l'Inde encouru par la diaspora indienne subalterne et la mobilisation de l'Inde dans les Antilles, mettant de nouveau l'accent sur l'importance primordiale des notions de la « femme indienne » dans les deux processus. L'autre fil conducteur du projet est l'importance de la musique populaire en tant que secteur clé de la politique culturelle. Tout en me concentrant sur le façonnement de la «femme indienne » et celui des identités « indiennes », je tente d'explorer un éventail de pratiques musicales à Trinité - des chutney, calypso et soca au chutney-soca, aux chansons de films en Hindi et à la rapso - afin de voir comment elles délimitent l'arène musicale où ces identités sont interprétées socialement.

L'argument central de ce projet est que les discours et pratiques de la modernité nationaliste ainsi que la formation de subjectivités modernes, en Inde et à Trinité peuvent être perçus différemment si l'on procède au repositionnement du cadre de l'analyse. Je suggère que ce repositionnement peut être effectué au moyen d'une double stratégie : l'étude des multiples processus par lesquels les migrants "subalternes » d'Inde sont désavoués que ce soit dans le passé ou au présent dans leur pays d'origine, et une étude des pratiques culturelles contemporaines des descendants de ces migrants dans leur pays actuel.

Pendant mes recherches à Trinité, je me suis passionnée pour les identités que l'on qualifiait « d'indiennes » aux Antilles. II était évident bien souvent que ceux qui participaient à la production de ces identités les percevaient comme partie intégrante d'une situation profondément « locale ", sans référence aucune à quelque lointain pays natal que ce soit. Alors que l'Inde au début du XXe siècle est engagée dans de nouvelles formes de mondialisation, quand j'ai entrepris mes recherches au début des années quatre-vingt-dix, ce processus n'était pas encore complètement engagé. Anticipant la formation de nouvelles hégémonies et m'interrogeant sur les interventions critiques pouvant être formulées, j'ai réfléchi à la première phase de mondialisation -celle qui avait été inspirée par l'Empire - pour retrouver la trace des migrations et de la transformation des corps et pratiques culturels et pour spéculer sur la formation du sujet que permettent le délogement et le relogement. Les déplacements 
géographiques et culturels, comme tend à le démontrer mon projet, ont formé des identités exclusivement locales dans les Antilles.

II a de cela quelques années, et alors que je rentrais de Trinité dans un avion de la BWIA de New York à Bombay, je me suis mise à bavarder avec une femme âgée afro-trinidadienne qui m'a demandé où j'allais. "En Inde » répondis-je. "Et pourquoi voulez-vous aller là-bas ? " me dit-elle. "Parce que j'y habite ». «Et comment cela se fait-il que vous viviez si loin de chez vous?"

近代的全球化进程时常将各个大陆之间以往历史联系的界线模糊化。最近我的 工作主要是研究东加勒比特尼达岛（Eastern Caribbean, in Trinidad）的印度裔居民 的日常文化实践。我认为, 身处印度的我们应当了解此地的贱民离散族群。这 些在1845至1917年期间被边缘化和排挤的劳工, 正和现代印度社会中那些不被 社会认可的印度人处于相似的地位。

以下是对这个研究项目中所提及的某些方法论和理论层面问题的综述。它指出 这样一种思考方式的意义所在, 那就是我们如何理解那些在我们自身所处的环 境之外，但却与其拥有相似的地缘政治位置的情境。

我将主要精力放在对跨南方情境的比较研究的框架进行理论化, 也致力于建立 习规之外的参照框架。这种方法广泛地跨越了各种学科, 涉及到文学研究、女 性主义理论、人类学、音乐学、电影研究等种种我用以进行文化生活研究的理 论。

这个研究项目中存在着一定的冲突, 于我而言形成了这样一种模糊的关系。这 种关系存在于二者之间。它们是: 一个地区一一加勒比地区, 这个地区起初对 我来说不过是一个民族志学概念上的研究对象, 同时, 在研究这个地区时, 我 还必须学会面对那些我在自身所处的后殖民语境中提出的种种批判后东方主义 的问题; 一个主题——“加勒比地区的印度人”，他们的历史乃至未来都可能 和我本人的大不相同。然而, 正如我曾经提出的那样, 考虑这个地区和这样一 个主题也同时可能改变身在印度的印度人对他们自身的文化身份问题的思考方 式。尽管在印度和加勒比地区之间存在着历史联系, 将详述于此的该比较研究 项目仍然必须面临这样的挑战, 它不仅应指出相似及相异之处, 还必须探讨地 点如何被对方定位。

我的研究项目首先希望做到的是替换西方现代性的这样一种地位: 在南部社会 分析中, 它是比较的唯一衡量标杆。我发现所有的学术研究都只是被定义为一 种比较研究, 实际上参照的隐性起点正是“西方”（虽然在各个语境下对“西 方” 有不同的想象)。因此, 在研究的开始, 我就提出应该在分析中建立习规 之外的其他参考框架，从而对抗这个比较性项目固有的不对等特性。

这个特定的任务曾经是对加勒比地区中对 “印度性”追寻表达过程的观察。这 些祈求曾经被从这样一个角度来审视, 这正是一个来自南亚次大陆, 跨越了半 
个地球，现在定居于哥伦布探险之旅的原目的地，被“发现”且被误命名的 （西）印度群岛的印度人的视角。在英帝国的故事中，在印度和加勒比地区 之间的历史联系常常被安排在书页留白处的注释中。我的研究项目的一个中心 问题就是: 对这些隐性联系的关注能够创造一种与那种关于印度和特尼达岛两 地现代性形成过程的习规叙述截然不同的叙事方式。虽然对这两个南方地理位 置中的“印度” 的追寻表达或是利用方式上显然存在着不同的历史叙事方式, 但我想说明的是，在审视这些历史的同时，我们可以用一种全新的方式来理解 这种想象的文化空间的意义和影响。

这个项目所致力于比较的重点在于妇女问题，且主要是对妇女问题这个范式的 表述和使用，而非仅仅在观察的层面上谈论妇女。我是从以下几个不同的角度 处理“印度妇女”这个概念的：其一，重新思考与二十世纪初期印度民族主义 运动相关的契约史（history of indentureship），从而探讨确认标准的印度妇女特 性的新视野; 其二, 将印度裔妇女视为一种令人振奋的加勒比地区新音乐的制 造者和表演者；其三，研究在“卡里普索（calypso）”和“索卡（soca）”这 两种音乐中，外来印度女性的历史发展和散布，或者是对在近代的特尼达岛文 化政治实践中外来印度女性形象进行重新审视; 其四，探讨特尼达的各类音乐 如何得益于印度的印地语电影行业。这一项目试图建立一种介于印度对其贱 民离散群体的否认与印度在加勒比（Caribbean）地区所做的动员之间的隐性联 系，同时再次强调在这两种过程中“印度妇女”概念的中心地位。

这个项目的另外一条线索是对作为文化政治实践的重要领地即流行音乐的关 注。一方面, 我继续关注“印度女性”的形成和“印度”身份的缔造, 同 时，我也致力于探讨在相当一个范围内特尼达音乐的运作情况，如从“楚特 尼 (chutney) ”、“卡利普索 (calypso) ”、“索卡(soca)” 到“楚特尼-索卡 (chutney-soca)”，印地语电影歌曲和“拉普索(rapso)” 这些种类，从而分析这些 音乐是如何在作为上述身份社会化表现的音乐圈中突现自我的。

这个研究计划的主要问题在于，在印度和特尼达岛两个语境之下，由于采用了 重新塑造的分析框架，对民族主义现代性的话语和实践，及现代主体性的形成 可能有完全不同的理解。我认为, 这种重新塑造能够通过一种双重策略而进 行。在这种双重策略中, 我们一方面可以开展一种对多元化进程的探讨, 在这 种多元化进程中来自印度的“贱民”移民们被排除于他们故国的历史和今天之 外; 另一方面, 我们可以对安居于现有家园的印裔移民所进行的近代文化实践 进行一次追问。

在特尼达岛作研究的时候, 我惊奇于在加勒比地区内被称为“印度人” 的各种 身份定义。通常来说，那些参与制造以上身份的人视它们为对值得自傲的“当 地” 情境的参予和归化, 而完全没有考虑到远方故土的影响。虽然在二十一世 纪初印度也同样参与了全球化的新形态，但在二十世纪九十年代初期我的研究 刚刚起步之时, 这种全球化进程还没有完全开展。我预测到了新的霸权形成过 程，思考过可能塑造何种批判介入的方式，也回顾了由英帝国所发起的全球化 的早期阶段，以追溯身体与文化习俗的迁移和转变，并且探讨这种解构和重构 的过程是以何种主要方式实现的。正如我的研究计划希望展示的那样, 地理和 历史的错位已经建立了种种加勒比地区特有的当地身份。

几年以前, 我从特尼达岛回印度的途中乘搭了一架从纽约飞往孟买的BWIA飞 机。在飞机上, 我和一位年长的非裔特尼达女士攀谈起来。她问我目的地是 哪里。我回答说是印度。她想知道为何我要去那里。我说我就住在印度。她问 道: “可是, 你怎么会离乡背井住在那么远的地方呢? ” 